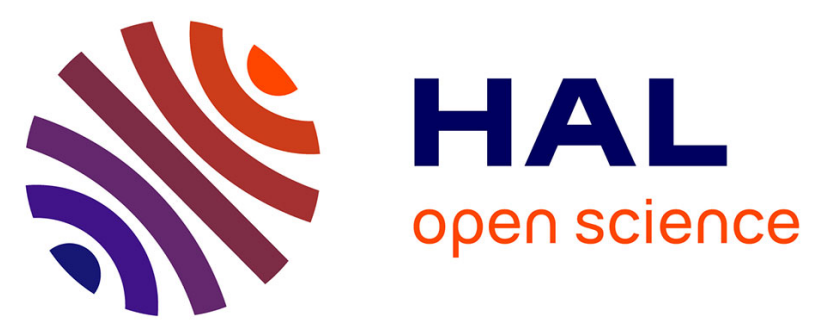

\title{
Irreversible decision making in contagious animal disease control under uncertainty: an illustration using FMD in Brittany
}

Olivier Mahul, Alexandre Gohin

\section{- To cite this version:}

Olivier Mahul, Alexandre Gohin. Irreversible decision making in contagious animal disease control under uncertainty: an illustration using FMD in Brittany. European Review of Agricultural Economics, 1999, 26 (1), pp.39-58. 10.1093/erae/26.1.39 . hal-01952087

\section{HAL Id: hal-01952087 \\ https://hal.science/hal-01952087}

Submitted on 3 Jun 2021

HAL is a multi-disciplinary open access archive for the deposit and dissemination of scientific research documents, whether they are published or not. The documents may come from teaching and research institutions in France or abroad, or from public or private research centers.
L'archive ouverte pluridisciplinaire HAL, est destinée au dépôt et à la diffusion de documents scientifiques de niveau recherche, publiés ou non, émanant des établissements d'enseignement et de recherche français ou étrangers, des laboratoires publics ou privés. 


\title{
Irreversible decision making in contagious animal disease control under uncertainty: an illustration using FMD in Brittany
}

\author{
Olivier Mahul and Alexandre Gohin
}

Institut National de la Recherche Agronomique, Rennes, France

\begin{abstract}
Summary
The concept of irreversible investment is applied to highly contagious animal disease control when uncertainty about the spread of the disease is resolved over time. In comparison with the strategy of destroying infected herds, the vaccination programme causes additional losses that cannot be recovered. These sunk costs are thus irreversible. Therefore, the gain from waiting for new information, namely the quasi-option value, should induce animal health authorities to delay the decision to vaccinate if the probability of a widespread epidemic is not too high. A numerical example is developed for foot and mouth disease (FMD) in Brittany.
\end{abstract}

Keywords: contagious animal disease, irreversible control strategy, foot and mouth disease, gain from waiting

JEL Classification: D81, Q10

\section{Introduction}

The livestock sector is exposed to highly contagious diseases, such as foot and mouth disease (FMD), which is one of the most feared. Cost-benefit analysis is frequently used in animal health economics to evaluate alternative strategies for contagious disease control. This economic evaluation is extensively based on expected values of gains and losses (Power and Harris, 1973; Berentsen et al., 1992; Garner and Lack, 1995), although the potential consequences of individual risk attitudes on the optimal dynamic decisionmaking process have recently been examined (Dijkhuizen et al., 1994; Mahul and Durand, 1998).

Animal epidemics are characterised by a low probability of occurrence and considerable potential losses affecting large numbers of herds. However, empirical data are typically scarce and therefore animal health authorities have little information about the effects of alternative control strategies on the spread of the disease and its economic consequences. The occurrence of a highly contagious disease creates a situation of Knightian uncertainty that cannot be described by an objective probability distribution. Nevertheless, uncertainty prevailing at a given time is resolved, at least partially, 
over time. The observed evolution of the disease allows animal health authorities to do a Bayesian updating of the prior distribution of potential losses.

In comparison with the control strategy consisting of destroying infected herds, a campaign of vaccination of susceptible herds should cause additional costs because of a decrease in value of vaccinated animals and intensified international trade restrictions. Therefore, this vaccination programme can be analysed as an investment generating costs that cannot be recovered. These sunk costs are thus irreversible (Pindyck, 1991). This decision can be postponed, giving animal health authorities the opportunity to wait for new information about the spread of the disease. This gain from waiting is nevertheless weighted against the cost of delaying the implementation of the irreversible vaccination policy.

In this paper, we examine how an irreversible strategy can affect the optimal dynamic decision-making process in contagious animal disease control under uncertainty. The discussion will begin with a short description of French policy on the control of FMD (Section 2). Using the literature on irreversible investment under uncertainty (Arrow and Fisher, 1974; Pindyck, 1991), we introduce into the standard epidemic model the gain from postponing the decision to vaccinate, called the quasi-option value, and the associated cost (Section 3). FMD outbreaks in France will be used as an illustration. We briefly present the simulation model of the economic consequences of FMD in France (Section 4) and then evaluate the quasi-option value of the vaccination strategy under several FMD scenarios (Section 5). Our main conclusion is that the resolution of uncertainty about the spread of the contagious disease should increase the propensity of animal health authorities to delay the decision to vaccinate. This entails that the strategy of late vaccination may be more efficient than the strategy of early vaccination if the subjective probability of a widespread epidemic occurring is not too high.

\section{French policy on the control of FMD}

FMD is a serious viral infection of domestic and wild cloven-hoofed animals. Cattle, sheep, goats and pigs are the species most commonly infected. The disease is characterised by development of vesicles in the mouth, on the muzzle, feet, teats and udder. Although the mortality rate is less than 6 per cent, except in young animals where it may exceed 50 per cent, the disease causes reduced milk yield and loss of weight (McCauley, 1979). Because of its ability to spread rapidly and to cause significant production losses, FMD is a major issue in international trade, and outbreaks can be associated with severe economic impacts as a result of loss of export markets. Countries or regions that are free from this disease include the 15 members of the European Union (EU), North and Central America, Australia, New Zealand and Japan.

As covered by the 1991 French law on FMD eradication, animal health authorities apply the strategy of stamping out (SO) if an FMD epidemic occurs in France. This would involve rigid quarantine of infected and suspect 
properties, prompt slaughter and disposal of animals infected with or exposed to FMD on infected premises, cleaning and disinfection of contaminated premises, and movement restrictions for livestock and livestock products in the control area that is established around the infected premises. This zone has an initial $10 \mathrm{~km}$ radius, which increases as the disease spreads. Animal health authorities could also undertake a campaign of vaccination coupled with the SO strategy. This entails that all the susceptible animals located in the control area and in a ring vaccination zone, a $5 \mathrm{~km}$ wide circular zone around the control area, would be vaccinated. The objective of vaccination is to dampen down the virus level circulating in the region and to reduce the risk of further spread (Donaldson and Doel, 1992).

French policy on the control of FMD has been affected by recent international initiatives regarding zoning in response to outbreaks of major animal diseases such as FMD. When an FMD outbreak is successfully contained within a quarantine zone, exports of livestock and livestock products from elsewhere in the country will remain unaffected, whereas the susceptible products coming from the infected region are excluded from international market until it recovers the status of FMD-free zone without vaccination. The period until the recognition of FMD freedom is (i) 3 months after the slaughter of the last infected herd when the SO strategy is implemented or (ii) 3 months after the slaughter of the last vaccinated herd if a campaign of vaccination is undertaken (Office International des Epizooties (OIE), 1993).

Therefore, implementing a campaign of vaccination coupled with the SO strategy generates additional costs that are sunk and thus largely irreversible: decrease in value of vaccinated livestock because of limited options for product disposal, and additional export losses caused by the delay necessary to slaughter the vaccinated animals.

\section{Flexibility, learning and irreversible control strategy}

To analyse how the possibility of waiting for new information about the dissemination of the contagious disease affects the optimal control strategy, we first study the disease dynamics in the standard epidemic model and then characterise the quasi-option value associated with the campaign of vaccination.

\subsection{Disease dynamics}

To study the effect of control strategies in disease dynamics, the standard epidemic model susceptible-infected-removed $(\mathrm{S}-\mathrm{I}-\mathrm{R})$ is used. This is a Markov process in which a closed population of size $n$ is subdivided into three classes consisting of those susceptible to the infection, those that are infected and those that are removed (Isham, 1993). As we focus on the economic consequences of the disease, this last health state is subdivided into two classes: immune through vaccination, and dead through stamping out or other non-disease causes. Herds are therefore classified into four health categories: susceptible, infected, immune, and dead, denoted by the 
set of health states $H \equiv\{s, i, m, d\}$. We denote the numbers of herds in these classes at date $t$ by $S_{t}, I_{t}, M_{t}$, and $D_{t}$, respectively, so that $S_{t}+I_{t}+M_{t}+D_{t} \equiv n$. We assume that $n \equiv 1$ without loss of generality. Thus $S_{t}, I_{t}$, and $M_{t}$ are the fractions of herds in health states $s, i$, and $m$ at date $t$. We refer to the fraction of infected herds $I_{t}$ as the prevalence of the disease. The fraction of herds in health state $h$ moving to state $h^{\prime}$ during period $t$ is denoted $\lambda_{h h^{\prime}}(t)$, where period $t$ starts at date $t-1$ and ends at date $t$.

When an animal epidemic occurs, health authorities always undertake the SO strategy. Its implementation is characterised in the epidemic model by the probability from state $i$ to state $d, \lambda_{i d}(t)$. The higher this probability, the more effective the strategy SO. The key transition rate of the epidemic model is the probability that a susceptible herd becomes infected in period $t, \lambda_{s i}(t)$. Because the disease is communicable, this probability is described as the product of the disease prevalence at date $t-1, I_{t-1}$, and the dissemination rate (DR) in period $t$, denoted $\beta_{t}$. The DR represents the average number of herds to which the disease agent is delivered by one affected herd, regardless of the state of the farm receiving the virus (Miller, 1979). Its size depends on environmental factors, type of farming, animal movements, farmer behaviour or disease control effects. Thus we can write

$$
\lambda_{s i}(t)=\beta_{t} I_{t-1} .
$$

Animal health authorities can also decide to apply a vaccination programme during period $t\left(v_{t}=1\right)$ or not $\left(v_{t}=0\right)$. In the first case, all the susceptible herds located in the control area and in the ring vaccination zone are vaccinated in one period. In addition, the vaccine is assumed completely effective and consequently provides a permanent protection. Therefore, the vaccinated herds move from state $s$ to state $m$. The irreversibility effect of the vaccination programme is thus due to the fact that the vaccinated herds have a permanent immunity against the disease.

The aggregate animal disease dynamics are characterised by the evolution of the number of herds in each health state through time with respect to the implemented control strategy. They are established by the following deterministic system:

$$
\begin{aligned}
S_{t+1} & =\left(1-\lambda_{s d}\right) S_{t}-\left(1-v_{t+1}\right) \beta_{t+1} I_{t} S_{t}-v_{t+1}\left(1-\lambda_{s d}\right) S_{t} \\
I_{t+1} & =\left(1-\lambda_{i d}\right) I_{t}+\left(1-v_{t+1}\right) \beta_{t+1} I_{t} S_{t} \\
M_{t+1} & =\left(1-\lambda_{m d}\right) M_{t}+v_{t+1}\left(1-\lambda_{s d}\right) S_{t} .
\end{aligned}
$$

This system is explained as follows. We assume that the transition rates are constant over time, except for the rate of contamination. The change in the fraction of susceptible herds is due to the exit of herds that become infected during the period $t+1, \beta_{t+1} I_{t} S_{t}$, or the exit of herds that become immune as a result of the campaign of vaccination, $\left(1-\lambda_{s d}\right) S_{t}$, and the exit generated by non disease-related mortality, $\lambda_{s d} S_{t}$. The change in the prevalence of infected herds arises from the entry of newly infected herds and the exit 
from the implementation of stamping out. If the SO strategy is effective, the transition probability $\lambda_{i d}$ is close to one. ${ }^{1}$ The change in the fraction of immune herds is caused by the entry of herds that are vaccinated. We assume there is no natural immunity, $\lambda_{i m}=0$, because herds are slaughtered before becoming immune. When the vaccination programme is undertaken in period $t$, the fraction of susceptible herds $S_{\tau}$, for all $\tau \geq t$, equals zero. Therefore, the fraction of immune herds is first equal to $\left(1-\lambda_{s d}\right) S_{t}$ and then it decreases at rate $\left(1-\lambda_{m d}\right)$. Throughout the analysis, we assume that the non-disease mortality rate is equal in health states $s$ and $m$, i.e. $\lambda_{s d}=\lambda_{m d}=\lambda$.

The main factor in the dynamics of the highly contagious disease is the DR. This epidemiological parameter is very sensitive, i.e. a marginal change may cause great changes in the spread of the disease and thus in the economic consequences. As with all catastrophic events, little information about its evolution over time is available. Nevertheless, the observed DRs provide useful information about the spread of the disease, which allows animal health authorities to forecast better the spread of the contagious disease and, therefore, to apply an appropriate control strategy.

\subsection{Learning and irreversible decision under uncertainty}

To analyse the irreversibility effect of the vaccination programme on the optimal dynamic decision-making process, we are considering a problem of sequential decisions in a two-period model. We denote by $y>0$ the net production of a susceptible herd per period, $l \in] 0, y]$ the production losses of an infected herd per period, $\left.l^{\prime} \in\right] 0, l[$ the production losses of an immune herd per period, $c$ the cost of vaccination per vaccinated herd and $F_{t}$ the fixed costs generated by the implementation of a vaccination programme at period $t$, for $t=1,2$. The later the vaccination strategy, the larger the number of vaccinated herds, the larger the delay to slaughter these herds and, therefore, the larger the losses as a result of import bans. Formally, this implies that $F_{1} \leq F_{2}$. These economic parameters are assumed to be known with certainty. The value of the production of an infected herd is therefore lower than for an immune herd, and the production of the slaughtered herd is reduced to zero. Animal health authorities are assumed to be risk-neutral and, therefore, the control strategy will be chosen to minimise the expected present losses. The equations derived in the current model are given in Appendix A.

Health authorities may apply a vaccination programme coupled with the SO strategy at period 1 or period 2 , named respectively early vaccination and late vaccination, or they may just undertake the SO strategy. Therefore, the decision-making process $\left(v_{1}, v_{2}\right)$ belongs to the following set:

$$
\left\{\left(v_{1}, v_{2}\right) / v_{1} \in\{0,1\} \text { and } v_{2} \in\left\{0,\left(1-v_{1}\right)\right\}\right\} \text {. }
$$

1 This transition rate is never equal to unity because some infected herds can excrete the virus, and thus contaminate susceptible herds, without having clinical signs. These different states of infection will be distinguished in the FMD simulation model. 
If animal health authorities decide to vaccinate the susceptible herds in the first period $\left(v_{1}=1\right)$, then no herd needs to be vaccinated in the second period $\left(v_{2}=0\right)$. On the contrary, if the vaccination programme is not undertaken in the first period $\left(v_{1}=0\right)$, then health authorities have the option of vaccinating the susceptible herds in the second period $\left(v_{2}=1\right.$ or $\left.v_{2}=0\right)$.

Uncertainty is introduced in the epidemic model through the DR. The DR in the first period is assumed to be known with certainty and it is equal to $\beta_{1}$, whereas the second-period DR $\tilde{\beta}_{2}$ is at present uncertain. This random variable is assumed to have a two-point support. Either the disease will be highly contagious, $\tilde{\beta}_{2}=\beta_{2}^{H}$, such that the late vaccination strategy is efficient with respect to the first-period DR under this widespread epidemic, or it will be weakly contagious, $\tilde{\beta}_{2}=\beta_{2}^{L}<\beta_{2}^{H}$, and as a consequence the SO strategy is efficient with respect to the first-period DR under this small epidemic. The policy maker evaluates the subjective probabilities of these two states as $p$ and $(1-p)$ respectively.

We define the critical second-period DR $\hat{\beta}_{2}$ such that animal health authorities are indifferent to undertaking the vaccination programme or not in period 2 when the SO strategy has been applied in period 1. It satisfies

$$
\begin{gathered}
l\left(1-\lambda_{i d}+\hat{\beta}_{2} S_{1}\right) I_{1}=l^{\prime}(1-\lambda) S_{1}+l\left(1-\lambda_{i d}\right) I_{1}+c S_{1}+F_{2} \\
\Leftrightarrow \hat{\beta}_{2}=\frac{1}{I_{1}} \frac{l^{\prime}(1-\lambda)+c+F_{2} / S_{1}}{l}
\end{gathered}
$$

with $S_{1}=\left(1-\lambda-\beta_{1} I_{0}\right) S_{0}$ and $I_{1}=\left(1-\lambda_{i d}+\beta_{1} S_{0}\right) I_{0}$.

Equation (4a) means that the expected losses when the SO strategy is applied in period 2 (LHS term) are equal to the expected losses when a campaign of vaccination is undertaken in period 2 (RHS term). The critical DR $\hat{\beta}_{2}$ decreases as the prevalence level $I_{1}$ and the per period losses of an infected herd $l$ increase, and this DR increases with the sunk costs caused by the vaccination programme $l^{\prime}, c$ and $F_{2}$. Therefore we assume ${ }^{2}$

$$
\beta_{2}^{L}<\hat{\beta}_{2}<\beta_{2}^{H} .
$$

Following Fisher and Hanemann $(1987,1990)$, we now consider two information scenarios. In the first one, uncertainty about the second-period DR is not resolved before the control strategy $v_{2}$ is chosen. In the second one, uncertainty is totally resolved by the beginning of period 2 (see Figures 1 and 2). Under these scenarios, the expected present losses over both periods are defined as a function of the first-period decision $v_{1} \in\{0,1\}$. The discount rate is assumed to be equal to zero without altering the results. These cost functions are respectively denoted $C^{*}\left(v_{1}\right)$ when uncertainty is not resolved, and $C^{* *}\left(v_{1}\right)$ when uncertainty is resolved.

2 If the DRs $\beta_{2}^{H}$ and $\beta_{2}^{L}$ are greater than the critical DR, $\hat{\beta}_{2}$, then implementing a campaign of vaccination is always optimal. On the contrary, if both DRs are lower than the critical DR, then applying a second-period vaccination strategy is never optimal. 
periods:

dates:

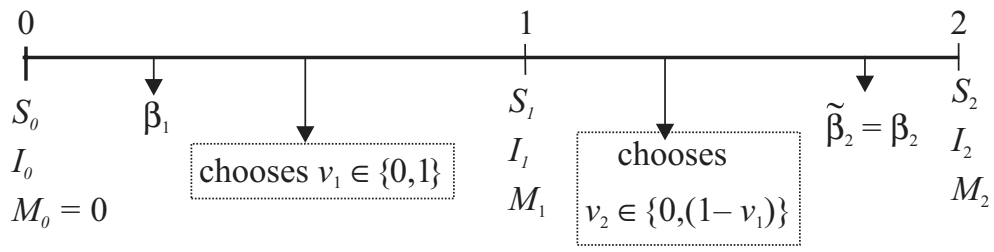

Figure 1. Decision process when uncertainty is not resolved before the second-period choice.

In the first scenario, the expected present losses are

$$
\begin{aligned}
C^{*}\left(v_{1}\right)= & l I_{1}+l^{\prime} M_{1}+\left(c S_{0}+F_{1}\right) v_{1} \\
& +\min _{v_{2} \in\left\{0,\left(1-v_{1}\right)\right\}} E\left[l \tilde{I}_{2}+l^{\prime} M_{2}+\left(c S_{1}+F_{2}\right) v_{2}\right] .
\end{aligned}
$$

The fractions of herds in health states are given by the epidemiological system (2) where the DRs are equal to the deterministic value $\beta_{1}$ in period 1 and the stochastic value $\tilde{\beta}_{2}$ in period 2 . When the campaign of vaccination is implemented in the first period, the cost function is

$$
C^{*}(1)=l^{\prime}(1-\lambda)(2-\lambda) S_{0}+l\left(1-\lambda_{i d}\right)\left(2-\lambda_{i d}\right) I_{0}+c S_{0}+F_{1}
$$

and if the SO strategy is undertaken in the first period, it satisfies

$$
C^{*}(0)= \begin{cases}l^{\prime}(1-\lambda) S_{1}+l\left(2-\lambda_{i d}\right) I_{1}+c S_{1}+F_{2} & \text { if } \bar{\beta}_{2} \geq \hat{\beta}_{2} \\ l\left(2-\lambda_{i d}+\bar{\beta}_{2} S_{1}\right) I_{1} & \text { if } \bar{\beta}_{2} \leq \hat{\beta}_{2}\end{cases}
$$

with $S_{1}=\left(1-\lambda-\beta_{1} I_{0}\right) S_{0}, I_{1}=\left(1-\lambda_{i d}+\beta_{1} S_{0}\right) I_{0}$ and $\bar{\beta}_{2}=p \beta_{2}^{H}+(1-p) \beta_{2}^{L}$.

The late vaccination programme is thus optimal if the expected secondperiod DR $\bar{\beta}_{2}$ is higher than the critical DR $\hat{\beta}_{2}$, and otherwise the SO strategy is efficient. As animal health authorities are assumed risk-neutral, this scenario is equivalent to the riskless problem where the second-period DR is $\bar{\beta}_{2}$. It can be shown that, under this scenario, the late vaccination strategy is dominated by the early vaccination strategy (see Appendix B). This

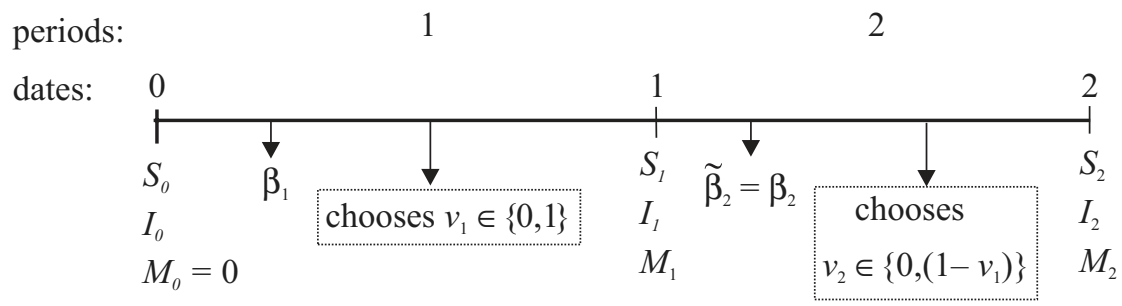

Figure 2. Decision process when uncertainty is resolved before the second-period choice. 
assertion may not hold when the decision maker receives more and better information about the spread of the disease.

In the second scenario, uncertainty is assumed to be totally resolved by the beginning of period 2. Animal health authorities can exploit this information before deciding to apply the vaccination programme. The expected present losses over both periods satisfy

$$
\begin{aligned}
C^{* *}\left(v_{1}\right)= & l I_{1}+l^{\prime} M_{1}+\left(c S_{0}+F_{1}\right) v_{1} \\
& +E\left\{\min _{v_{2} \in\left\{0,\left(1-v_{1}\right)\right\}}\left[l \tilde{I}_{2}+l^{\prime} M_{2}+\left(c S_{1}+F_{2}\right) v_{2}\right]\right\} .
\end{aligned}
$$

The campaign of vaccination undertaken in period 2, given that this strategy is not applied in period 1 , is socially efficient only if $\tilde{\beta}_{2}=\beta_{2}^{H}$, and otherwise the SO strategy is optimal. We have thus

$$
C^{* *}(1)=C^{*}(1)
$$

and

$$
\begin{aligned}
C^{* *}(0)= & l I_{1}+p\left\{l^{\prime}(1-\lambda) S_{1}+l\left(1-\lambda_{i d}\right) I_{1}+c S_{1}+F_{2}\right\} \\
& +(1-p)\left\{l\left(1-\lambda_{i d}+\beta_{2}^{L} S_{1}\right) I_{1}\right\}
\end{aligned}
$$

with $S_{1}=\left(1-\lambda-\beta_{1} I_{0}\right) S_{0}$ and $I_{1}=\left(1-\lambda_{i d}+\beta_{1} S_{0}\right) I_{0}$.

The gain from being able to learn about the future spread of the disease, called the quasi-option value of the vaccination programme, is thus expressed in our model by

$$
Q=C^{*}(0)-C^{* *}(0)
$$

and, from equations (7b) and (9b), we obtain

$$
Q= \begin{cases}(1-p) S_{1}\left[l^{\prime}(1-\lambda)+c+F_{2} / S_{1}-l \beta_{2}^{L} I_{1}\right] & \text { if } \bar{\beta}_{2} \geq \hat{\beta}_{2} \\ -p S_{1}\left[l^{\prime}(1-\lambda)+c+F_{2} / S_{1}-l \beta_{2}^{H} I_{1}\right] & \text { if } \bar{\beta}_{2} \leq \hat{\beta}_{2} .\end{cases}
$$

The quasi-option value can be rewritten in terms of the subjective probability $p$ as

$$
Q= \begin{cases}(1-p) S_{1}\left[l^{\prime}(1-\lambda)+c+F_{2} / S_{1}-l \beta_{2}^{L} I_{1}\right] & \text { if } p \geq \hat{p} \\ p S_{1}\left[l \beta_{2}^{H} I_{1}-l^{\prime}(1-\lambda)-c-F_{2} / S_{1}\right] & \text { if } p \leq \hat{p}\end{cases}
$$

with the critical probability $\hat{p}=\left(\hat{\beta}_{2}-\beta_{2}^{L}\right) /\left(\beta_{2}^{H}-\beta_{2}^{L}\right)$.

Given assumption (5), $Q$ is unambiguously positive. When the probability $p$ is higher than the critical probability $\hat{p}, Q$ increases as the sunk costs of vaccination increase, and as the prevalence level $I_{1}$ and the per period production loss of an infected herd decrease. When $p \leq \hat{p}, Q$ increases as the per period loss of an infected herd and the prevalence level increase, and as the per period loss of an immune herd and the sunk costs of vaccination decrease. The quasi-option value $Q$ is single peaked with $Q$ increasing (decreasing) for $p<\hat{p}(p>\hat{p})$. It reaches a maximum at $p=\hat{p}$, in which case the two expressions in (12) are identical. 
The irreversibility effect of the vaccination programme is therefore based upon two opposite effects. On the one hand, the costs caused by this control strategy are sunk and thus irreversible. Delaying this strategy allows animal health authorities to save these sunk costs if the epidemic turns out to be small. This informational gain is measured by the quasi-option value $Q$. On the other hand, delaying the decision to vaccinate may cause additional cost $\left[C^{*}(0)-C^{*}(1)\right]$, which is also irreversible. Therefore, the optimal control strategy depends on the comparison of these two effects.

Implementing the vaccination programme in the first period, namely the 'act then learn' strategy, is thus optimal if the cost of waiting is larger than the gain from waiting, i.e. $C^{*}(0)-C^{*}(1) \geq Q$. This means that animal health authorities apply the 'precautionary principle'. On the contrary, postponing the decision to vaccinate, namely the 'learn then act' strategy, is optimal if $C^{*}(0)-C^{*}(1) \leq Q$. In this case, the late vaccination programme will be undertaken if $\tilde{\beta}_{2}=\beta_{2}^{H}$, and the early vaccination campaign is thus dominated by the late one. It is of interest that if the early vaccination programme is optimal when uncertainty is totally resolved, then it is optimal when uncertainty is not resolved because $Q$ is always positive.

To investigate the impact on the optimal control strategy of epidemiological and economic parameters, we have recourse to comparative static analysis.

As $\left[C^{* *}(0)-C^{* *}(1)\right]$ increases with $F_{2}$ when $\bar{\beta}_{2} \geq \hat{\beta}_{2}$, so also do the fixed losses caused by the late vaccination programme and the propensity to undertake the early vaccination programme. Furthermore, if the critical second-period DR, $\hat{\beta}_{2}$, is larger than the DR, $\beta_{2}^{H}$, then the late vaccination programme is never optimal. This entails that the larger the incubation period of the disease, the later the resolution of uncertainty, and the weaker the propensity to delay the irreversible decision to vaccinate.

$\left[C^{*}(0)-C^{*}(1)\right]$ is shown to be increasing with $\beta_{2}^{H}$ if $\bar{\beta}_{2} \leq \hat{\beta}_{2}$. This means that the animal health authorities are induced to undertake the early vaccination programme when uncertainty is not resolved. The quasi-option value $Q$ also increases with $\beta_{2}^{H}$ when $\bar{\beta}_{2} \leq \hat{\beta}_{2}$. In addition, one can easily show that these marginal changes are identical. This means that a change in the DR $\beta_{2}^{H}$ does not affect the optimal decision when uncertainty is totally resolved.

When the low second-period DR, $\beta_{2}^{L}$, increases, $\left[C^{*}(0)-C^{*}(1)\right]$ increases if $\bar{\beta}_{2} \leq \hat{\beta}_{2}$ and otherwise it is invariant, and $Q$ decreases if $\bar{\beta}_{2} \geq \hat{\beta}_{2}$ and otherwise it is invariant. Consequently, the higher $\beta_{2}^{L}$, the higher the propensity to undertake the early vaccination programme when uncertainty is totally resolved.

It follows that the larger the spread between $\beta_{2}^{H}$ and $\beta_{2}^{L}$, the more uncertain the FMD spread and, therefore, the larger the quasi-option value. It should be noted that if changes in DRs are such that they are both under or above the critical DR $\hat{\beta}_{2}$, then the quasi-option value becomes null.

All these findings are illustrated through simulated FMD outbreaks in Brittany. 


\section{Simulation model of FMD losses in France}

The modelling approach includes two sub-models. The epidemiological model simulates the spread of the disease with respect to the control strategy implemented by the animal health authorities. The economic model uses the epidemiological results to estimate the direct and indirect economic impacts on the affected region and on the French economy as a whole (Mahul and Durand, 1998).

The epidemiological model generates outbreak scenarios and defines how the FMD epidemic is likely to behave (see Figure 3). It is based upon a deterministic state-transition model developed from a Markov chain. The time step is half a week and the herds are the unit of concern. The standard $\mathrm{S}-\mathrm{I}-\mathrm{R}$ model is decomposed according to the method of stages (Isham, 1993). First, the health state susceptible is subdivided into two stages. Susceptible herds are exposed to contamination in the control area and they are unexposed to contamination when they are outside. Moving between these two states is due to the expansion of the control area as the FMD epidemic spreads. Second, the period of infection is subdivided into three stages. The incubation period is the time from exposure until the affected herd infects others. The invasion period is defined as the period during which the affected herd excretes the virus and contaminates susceptible herds without having clinical signs. The clinical period is the time from which the affected herd has clinical signs until it is slaughtered. Herds are therefore divided into seven health categories: susceptible and non-exposed to contamination, susceptible and exposed to contamination through the expansion of the control area, incubation stage, invasion stage, clinical stage, immune through vaccination and dead through stamping out. The probability that a vaccinated herd becomes immune in one period is assumed to be equal to 0.9 , contrary to the previous theoretical model where this probability was implicitly assumed to equal unity. Consequently, the vaccination programme lasts more than one period and it is carried on until all the susceptible herds are vaccinated.

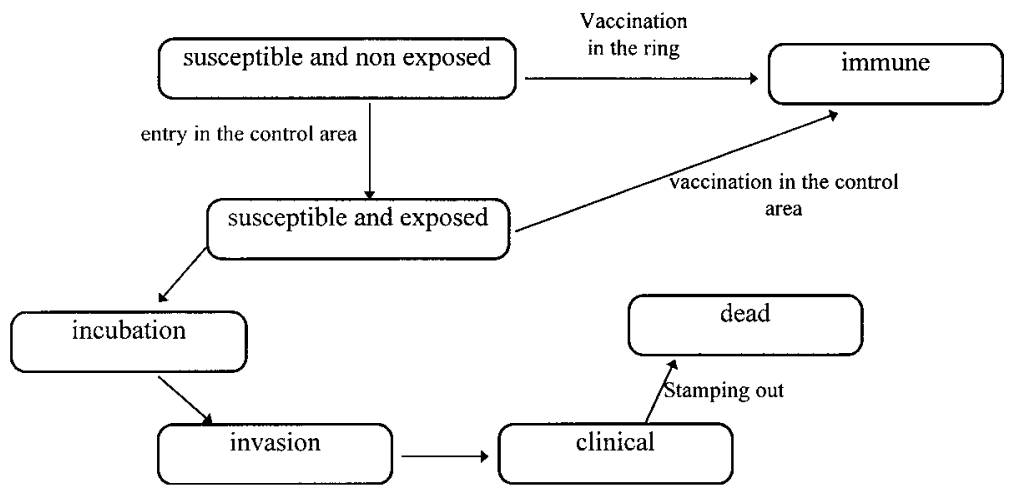

Figure 3. Pathways considered in FMD state-transition model. 
From these epidemiological results, the economic model estimates the impacts on regional and national economies. The estimated losses caused by the FMD epidemic are divided into direct costs and indirect costs. Direct costs are divided into (i) disease-control costs and costs of vaccination, (ii) compensation payments payable for direct losses of livestock that are slaughtered through the control strategy, (iii) incidental costs on non-infected farms and in the control area as a result of movement restrictions, (iv) decrease in value for vaccinated animals because of limited options of product disposal and (v) incidental costs on meat manufacturing and milk manufacturing sectors. The direct costs (i) and (ii) are borne by the French government (40 per cent) and by the European Union (60 per cent).

The indirect losses borne by the French economy are caused by the international trade restrictions. They depend upon the livestock and livestock products subject to import bans, the duration and the region under these bans. Products which may be subject to import bans are gathered into eight categories: beef/veal and breeding cattle; pigmeat and breeding pigs; sheep/ goat meat and breeding sheep and goats; milk; butter and cream; cheese; condensed milk; and other dairy products. Among importing countries, the states of the European Union and the non-EU countries are distinguished. Therefore, the economic model allows us, for each category of products and each group of countries, to define the type of import ban during the epidemic (national, regional, none), the duration of the import ban after the eradication of the disease from the affected region and the country. The consequences of import bans on French economic sectors are assessed through a multisectoral analysis that contributes to evaluating the changes in the exports of a given sector on the French economy as a whole. Such an analysis is based on an input-output model (Mahul and Durand, 1998).

This simulation model is used to estimate the quasi-option values of the vaccination strategy under different scenarios when FMD outbreaks occur in Brittany. ${ }^{3}$

\section{Estimates of quasi-option values for FMD outbreaks in Brittany}

FMD outbreaks are simulated in Brittany, which is one of the major livestock production regions in France. The livestock industries consist mainly of dairy cattle and intensive pig farming. The number of cattle farms and pig farms is respectively 25,000 and 11,000 , which rear 2.4 million cattle and 7.4 million pigs. Of these figures, 54 per cent of French pig farms and 13 per cent of French dairy farms are located in Brittany. Agriculture accounts for 7.4 per cent of the regional gross domestic product. Brittany exports pork, beef

3 These simulations are carried out with a computer model developed for the French Ministry of Agriculture. It allows the decision maker to assess the direct and indirect economic consequences of FMD outbreaks and, therefore, to select the optimal control strategy with respect to an economic criterion. 
and dairy products representing respectively 45 per cent, 13 per cent and 8 per cent of French exports.

We assume that recent international initiatives regarding zoning in response to FMD outbreaks and the delays until freedom from FMD is recognised are observed by all the importing countries. ${ }^{4}$ This means that the economic consequences of this FMD epidemic affect only Brittany. If the vaccination strategy is applied, vaccinated animals are assumed to be slaughtered in a period of 2 months after the eradication of the disease. Therefore, import bans are maintained 3 months after the eradication of the FMD epidemic if the SO strategy is applied, and 5 months after the eradication of the FMD epidemic if the vaccination campaign is undertaken. The set of products that are subject to import bans are beef and breeding cattle, pork and breeding pigs, sheep meat and breeding sheep, goat meat and breeding goats, and goat milk.

One pig farm is assumed to be infected at the start of the simulation. Given the previous study on FMD dynamics in France (Mahul and Durand, 1998), we will consider the following two scenarios of FMD dissemination. Under the optimistic scenario, animal health authorities are able to limit the FMD spread, so that the vector of DRs per period is $\beta^{L}=$ $\{9.32 ; 1.04 ; 0.30 ; 0.30 \ldots\}$. Under the pessimistic scenario, they have some difficulties eradicating the disease and, therefore, the vector of DRs per period is $\beta^{H}=\{9.32 ; 1.04 ; 0.48 ; 0.48 \ldots\}$. The DRs are assumed known with certainty during the two first periods, i.e. during the first week, with a considerable downward jump between the first DR and the second one. Uncertainty about the FMD dynamics is thus totally resolved during the second week.

The animal health authorities undertake a strategy of stamping out (SO), which can be coupled with an early vaccination programme applied in the first week (SOEV) or with a late vaccination programme implemented in the second week (SOLV).

The epidemiological and economic impacts of these two strategies are simulated and the results are shown in Appendix C.

Under the optimistic scenario, where $\beta_{2}^{L}=0.30$, the SO strategy turns out to be the least expensive. The FMD epidemic lasts 11.5 weeks after detection and involves 222 affected herds, which means that 68,500 animals would be slaughtered. The direct costs are 318 million French francs (mFF) of which 31 per cent are subsidised by the European Union. The regional losses, which include the direct costs and the indirect costs borne by the regional sectors, are $839 \mathrm{mFF}$ for the breeding sector and $2229 \mathrm{mFF}$ for the Breton economy as a whole. If the SOEV strategy were carried out, these losses would be $2859 \mathrm{mFF}$, i.e. 28 per cent higher than under the SO strategy. It should be noted that the programme of late vaccination SOLV is more expensive than that of early vaccination $\operatorname{SOEV}(+8$ per cent). 


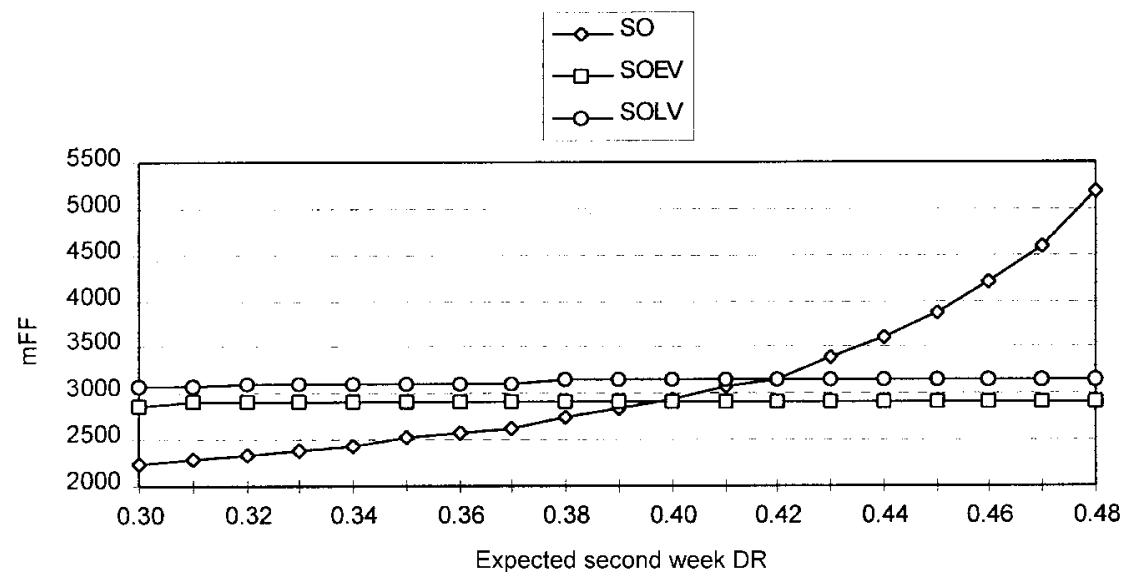

Figure 4. Losses borne by the Breton economy with respect to the expected secondweek dissemination rate (DR), by control strategy.

Under the pessimistic scenario, where $\beta_{2}^{H}=0.48$, the SOEV strategy is optimal. The FMD epidemic lasts 6 weeks, 187 herds are infected and 1406 herds are vaccinated. The total losses borne by the Breton economy are $2912 \mathrm{mFF}$. They would increase by 8 per cent if the SOLV strategy were applied and by 78 per cent if the SO strategy were carried out. The SOEV strategy would result in a much shorter duration of the epidemic with respect to the SO strategy, which would offset the additional delay of import bans in order to slaughter the vaccinated animals.

The expected second-week DR $\bar{\beta}_{2}=p \beta_{2}^{H}+(1-p) \beta_{2}^{L}$ is computed from the decision maker's subjective probability $p \in[0,1]$ of the pessimistic scenario occurring. We assume that animal health authorities select the control strategy to minimise the net expected losses borne by the Breton economy as a whole. These regional costs under each control strategy are shown in Figure 4. Under the SO strategy, the regional costs vary from $2229 \mathrm{mFF}$ to $5194 \mathrm{mFF}$. The losses caused by the SOEV and SOLV strategies are globally constant and they are respectively equal to $2900 \mathrm{mFF}$ and $3100 \mathrm{mFF}$. The critical second-week DR $\hat{\beta}_{2}$ is thus equal to 0.42 . This means that the regional losses as a result of the SO strategy are larger (smaller) than those caused by the SOLV strategy if the expected second-week DR $\bar{\beta}_{2}$ is higher (lower) than the critical DR $\hat{\beta}_{2}$. From equation (10), the quasi-option value $Q$ of the irreversible vaccination programme is expressed as a function of the probability $p$. This gain from waiting for new information is then compared with the cost of delaying the decision $\left[C^{*}(0)-C^{*}(1)\right]$. As we have noticed in the theoretical model, the quasi-option value increases with $p \leq \hat{p}$ where $\hat{p}=0.65$ in our illustration (see Figure 5), and then it decreases. Its maximum level is $332 \mathrm{mFF}$, which equals 11.4 per cent of the losses caused by the SOEV strategy. The cost of waiting increases with $p$ until $p=\hat{p}$ and then it is constant and equal to $235 \mathrm{mFF}$. 


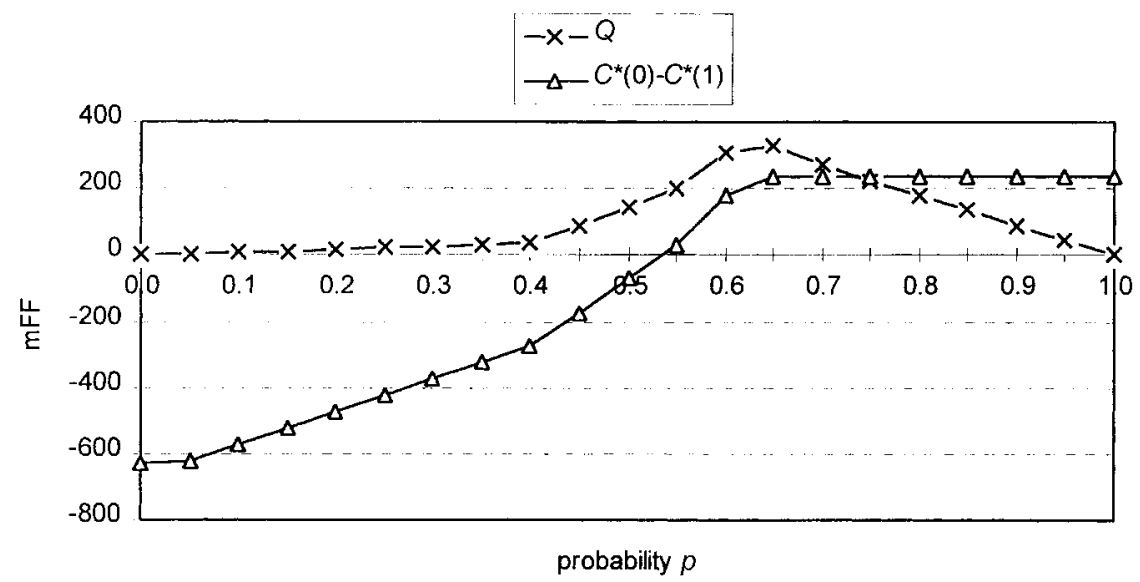

Figure 5. Gain from waiting for new information $Q$ and cost of delaying the irreversible vaccination programme $\left[C^{*}(0)-C^{*}(1)\right]$, with $\beta_{2}^{L}=0.30$ and $\beta_{2}^{H}=0.48$.

If uncertainty is not resolved before making the second-week decision, then the SOEV strategy is applied if $\left[C^{*}(0)-C^{*}(1)\right] \geq 0$, i.e. if the subjective probability $p$ is higher than the critical probability $p^{*}=0.55$ (see Figure 5), and the SO strategy is otherwise undertaken. In this case, delaying the vaccination programme is never optimal because it only generates additional costs. If uncertainty is totally resolved, the SOEV strategy is optimal if and only if the cost of waiting is higher than the quasi-option value $Q$, i.e. if the subjective probability $p$ is higher than the critical probability $p^{* *}=0.75$. Therefore, the resolution of uncertainty increases the critical probability from which the SOEV strategy is optimal. This entails that if the subjective probability $p$ is in the interval $[0.55 ; 0.75]$, the SOEV strategy is optimal when uncertainty is not resolved, whereas it is inefficient when uncertainty is totally resolved. Consequently, the SOLV strategy is optimal if the pessimistic scenario occurs, whereas the SO strategy is optimal under the optimistic scenario.

The optimal control strategy is also altered by the values of low and high second-week DRs. To illustrate this fact, we consider three scenarios that yield the same expected second-week DR, $\bar{\beta}_{2}=0.43$. In Option I, the second-week DR is equal to $\beta_{2}^{L}=0.30$ or $\beta_{2}^{L}=0.48$. Animal health authorities expect that a widespread epidemic will occur with probability $p=0.72$. In option II, the low second-period DR increases to 0.40 and the probability of widespread outbreak is $p=0.38$. In option III, the high second-week DR decreases to 0.45 and the probability of widespread outbreak is $p=0.87$. Therefore, uncertainty about the disease dynamics is the most important under option I. Simulations are shown in Table 1. If uncertainty about the spread of FMD is not resolved, which means that the quasi-option value is null, then it is decided to carry out the SOEV strategy because $\left[C^{*}(0)-C^{*}(1)\right]$ is positive. Let us assume now that uncertainty is totally resolved before choosing the second-week strategy. Under option I, the 
Table 1. Gain of waiting $Q$ and cost of waiting $\left[C^{*}(0)-C^{*}(1)\right]$ with respect to secondweek DRs, with $\bar{\beta}_{2}=0.43$

\begin{tabular}{llll}
\hline & $\begin{array}{l}\text { Option I } \\
\beta_{2}^{L}=0.30, \beta_{2}^{H}=0.48, \\
p=0.72\end{array}$ & $\begin{array}{l}\text { Option II } \\
\beta_{2}^{L}=0.40, \beta_{2}^{H}=0.48, \\
p=0.38\end{array}$ & $\begin{array}{l}\text { Option III } \\
\beta_{2}^{L}=0.30, \beta_{2}^{H}=0.45, \\
p=0.87\end{array}$ \\
\hline$Q(\mathrm{mFF})$ & 252 & 126 & 117 \\
{$\left[C^{*}(0)-C^{*}(1)\right](\mathrm{mFF})$} & 235 & 235 & 235 \\
\hline
\end{tabular}

quasi-option value is $252 \mathrm{mFF}$. It is larger than the cost of waiting $\left[C^{*}(0)-C^{*}(1)\right]$ and, therefore, it is optimal to delay the decision to vaccinate. Under option II, the quasi-option value $Q$, which equals $126 \mathrm{mFF}$, is lower than the cost of waiting and, therefore, applying the SOEV strategy remains optimal even if uncertainty is resolved. The same results are obtained under option III. As noticed in the theoretical model, reducing the spread between the second-week DRs, which is the case in options II and III, generates a reduction of uncertainty, which yields a decrease of $Q$.

\section{Conclusions}

The concept of irreversibility has been applied to contagious animal disease control under uncertainty. We have first developed a theoretical framework based upon the standard epidemiological model $\mathrm{S}-\mathrm{I}-\mathrm{R}$ in which the gain from waiting for new information about the disease spread, namely the quasi-option value, has been introduced. Delaying the decision to vaccinate is thus optimal if the quasi-option value is larger than the cost of waiting. In this case, the 'learn then act' strategy prevails. On the contrary, the early vaccination strategy is optimal if the quasi-option value is lower than the cost of waiting and, therefore, the decision maker applies the 'precautionary principle'.

A simulation model of FMD epidemic in Brittany is used as an illustration. A small epidemic would cause losses of $2229 \mathrm{mFF}$ borne by the Breton economy as a whole if the strategy of stamping out were applied, and a widespread epidemic would cause losses of $2912 \mathrm{mFF}$ if the early vaccination strategy were implemented. The maximum quasi-option value is estimated at $322 \mathrm{mFF}$. Our results suggest that applying the early vaccination campaign is optimal if the animal health authorities consider that the probability of a large epidemic is higher than 55 per cent when uncertainty about the disease spread is not resolved, and 75 per cent when uncertainty is totally resolved. Therefore, the resolution of uncertainty reduces the propensity to undertake this early vaccination strategy.

Nevertheless, these empirical results are regionally dependent because they are based upon epidemiological and economic factors that vary strongly according to region. Previous simulations have shown that Brittany may be the only French region where the campaign of vaccination could be 
optimal under reasonable assumptions (Mahul and Durand, 1998). For example, if FMD outbreaks occur in the Provence-Alpes-Côte d'Azur region of southeastern France then, under the pessimistic scenario where $\beta^{H}=\{6.47 ; 2.20 ; 0.48 ; 0.48 \ldots\}$, the total losses borne by the regional economy should be equal to $66 \mathrm{mFF}$ under the SO strategy, $132 \mathrm{mFF}$ under the SOEV strategy and $135 \mathrm{mFF}$ under the SOLV strategy. Implementing the strategy of stamping out is thus less than half as expensive as undertaking a campaign of early vaccination.

This article has emphasised the impact of information in selecting a public control strategy for eradicating FMD outbreaks. Its objective is not to support real-life decision making, but only to point out that not integrating this informational parameter into the dynamic decision-making process may lead animal health authorities to undertake the early vaccination programme more often than they should. The irreversible effect has been highlighted in a two-state model where uncertainty was totally resolved in one period. A more realistic approach should consider this issue in a multiperiod stochastic model where active learning generated by the observations on the state of the epidemic disease would convey information about uncertainty and, therefore, it would contribute to resolving uncertainty over time. This analysis seems all the more important as selecting an inadequate control strategy may cause large additional losses. Further research should expand this framework to other highly contagious diseases such as swine fever.

\section{Acknowledgements}

We wish to thank the three anonymous ERAE referees and the Editor for their constructive comments on an earlier draft of this paper. The first author acknowledges financial support from the Bureau de l'Epidémiologie et des Prophylaxies en Elevages de Ruminants, Direction Générale de l'Alimentation, France.

\section{References}

Arrow, K. J. and Fisher, A. C. (1974). Environmental preservation, uncertainty, and irreversibility. Quarterly Journal of Economics 88: 312-319.

Berentsen, P. B., Dijkhuizen, A. A. and Oskam, A. J. (1992). A dynamic model for costbenefit analyses of foot-and-mouth disease control strategies. Preventive Veterinary Medicine 12: 229-243.

Dijkhuizen, A. A., Hardaker, J. B. and Huirne, R. B. (1994). Risk attitude and decision making in contagious disease control. Preventive Veterinary Medicine 18: $203-212$.

Donaldson, A. I. and Doel, T. R. (1992). Foot-and-mouth disease: the risk for Great Britain after 1992. Veterinary Record 131: 114-120.

Fisher, A. C. and Hanemann, W. M. (1987). Quasi-option value: some misconceptions dispelled. Journal of Environmental Economics and Management 14: 183-190.

Fisher, A. C. and Hanemann, W. M. (1990). Option value: theory and measurement. European Review of Agricultural Economics 17: 167-180.

Garner, M. G. and Lack, M. B. (1995). An evaluation of alternate control strategies for footand-mouth disease in Australia: a regional approach. Preventive Veterinary Medicine 23: 9-32. 
Isham, V. (1993). Stochastic models for epidemics with special reference to AIDS. Annals of Applied Probability 3(1): 1-27.

Mahul, O. and Durand, B. (1998). Economic consequences of the FMD epidemics and their public control in France. Unpublished paper. INRA Rennes.

McCauley, E. H. (1979). Estimation of the physical loss in animals infected with foot-andmouth disease. In E. H. McCauley, N. A. Aulaqi, J. C. New, W. B. Sunquist and W. M. Miller (eds), Potential Economic Impact of Foot-and-Mouth Disease in the United States. University of Minnesota, 23-32.

Miller, W. M. (1979). A state-transition model of epidemic foot-and-mouth disease. In E. H. McCauley, N. A. Aulaqi, J. C. New, W. B. Sunquist and W. M. Miller (eds), Potential Economic Impact of Foot-and-Mouth Disease in the United States. University of Minnesota, 56-72.

Office International des Epizooties (OIE) (1993). International Animal Health Code, 6th edn. Paris: OIE.

Pindyck, R. S. (1991). Irreversibility, uncertainty, and investment. Journal of Economic Literature 29: 1110-1148.

Power, A. P. and Harris, S. A. (1973). A cost-benefit evaluation of alternative control policies for foot-and-mouth disease in Great Britain. Journal of Agricultural Economics 24: $573-597$.

\section{Appendix A}

\section{Proof of equations (4)}

When animal health authorities do not undertake the vaccination programme in period 2, the losses in period 2 are caused only by the infected herds

$$
l I_{2}=l\left[\left(1-\lambda_{i d}\right) I_{1}+\hat{\beta}_{2} I_{1} S_{1}\right]=l\left(1-\lambda_{i d}+\hat{\beta}_{2} S_{1}\right) I_{1}
$$

where the prevalence of the disease in period $2, I_{2}$, is expressed in terms of the fractions of susceptible herds and infected herds in period 1 through equation (2b) with $v_{2}=0$.

When animal health authorities decide to implement the vaccination strategy in period 2, the losses in period 2 are due to the vaccinated herds, the herds infected during the previous period and the vaccination costs

$$
l^{\prime} M_{2}+l I_{2}+c S_{1}+F_{2}=l^{\prime}(1-\lambda) S_{1}+l\left(1-\lambda_{i d}\right) I_{1}+c S_{1}+F_{2}
$$

by using respectively equation (2c) with $M_{1}=0$, as the vaccination programme was not undertaken during the first period, and $v_{2}=1$, and equation ( $2 \mathrm{~b}$ ) with $v_{2}=1$. Comparing equations (A1) and (A2) yields the critical second-period DR expressed in equation (4b).

\section{Proof of equations (7)}

In the first scenario, the expected present losses caused by the implementation of the vaccination programme in the first period are

$$
C^{*}(1)=l I_{1}+l^{\prime} M_{1}+c S_{0}+F_{1}+l E \tilde{I}_{2}+l^{\prime} M_{2}
$$

with

$$
\begin{aligned}
I_{1} & =\left(1-\lambda_{i d}\right) I_{0} \\
M_{1} & =(1-\lambda) S_{0} \\
M_{2} & =(1-\lambda) M_{1}=(1-\lambda)^{2} S_{0} \\
E \tilde{I}_{2} & =\left(1-\lambda_{i d}\right) I_{1}=\left(1-\lambda_{i d}\right)^{2} I_{0}
\end{aligned}
$$

where equations (A4)-(A7) are deduced from the deterministic system (2) with $v_{1}=1$ and 
$v_{2}=0$. Integrating equations (A4) $-(\mathrm{A} 7)$ in equation (A3) yields

$$
\begin{aligned}
C^{*}(1) & =l^{\prime}\left[(1-\lambda)+(1-\lambda)^{2}\right] S_{0}+l\left[\left(1-\lambda_{i d}\right)+\left(1-\lambda_{i d}\right)^{2}\right] I_{0}+c S_{0}+F_{1} \\
& =l^{\prime}(1-\lambda)(2-\lambda) S_{0}+l\left(1-\lambda_{i d}\right)\left(2-\lambda_{i d}\right) I_{0}+c S_{0}+F_{1} .
\end{aligned}
$$

If the vaccination strategy is not undertaken in the first period, i.e. $v_{1}=0$ and consequently $M_{1}=0$, it is implemented in period 2 if and only if $\bar{\beta}_{2} \geq \hat{\beta}_{2}$. From this, the two following cases are derived.

When $\bar{\beta}_{2} \leq \hat{\beta}_{2}$, the expected present losses are

$$
C^{*}(0)=l I_{1}+l E \tilde{I}_{2}
$$

with

$$
E \tilde{I}_{2}=\left(1-\lambda_{i d}\right) I_{1}+\bar{\beta}_{2} S_{1} I_{1}=\left(1-\lambda_{i d}+\bar{\beta}_{2} S_{1}\right) I_{1}
$$

which is derived from equation (2b) with $v_{1}=v_{2}=0$. We thus obtain

$$
C^{*}(0)=l\left(2-\lambda_{i d}+\bar{\beta}_{2} S_{1}\right) I_{1} .
$$

When $\bar{\beta}_{2} \geq \hat{\beta}_{2}$, the expected present losses are

$$
C^{*}(0)=l I_{1}+l E \tilde{I}_{2}+l^{\prime} M_{2}+c S_{1}+F_{2}
$$

with

$$
\begin{aligned}
& E \tilde{I}_{2}=\left(1-\lambda_{i d}\right) I_{1} \\
& M_{2}=(1-\lambda) S_{1}
\end{aligned}
$$

which are derived from equations (2) with $v_{1}=0$ and $v_{2}=1$. Integrating equations (A13) and (A14) in equation (A12) yields

$$
\begin{aligned}
C^{*}(0) & =l I_{1}+l\left(1-\lambda_{i d}\right) I_{1}+l^{\prime}(1-\lambda) S_{1}+c S_{1}+F_{2} \\
& =l^{\prime}(1-\lambda) S_{1}+l\left(2-\lambda_{i d}\right) I_{1}+c S_{1}+F_{2} .
\end{aligned}
$$

\section{Proof of equation (11)}

The quasi-option value of the vaccination programme expressed in equation (10) is reformulated using equations ( $7 b)$ and $(9 b)$.

When $\bar{\beta}_{2} \leq \hat{\beta}_{2}$, it satisfies

$$
\begin{aligned}
Q= & {\left[l I_{1}+l\left(1-\lambda_{i d}\right) I_{1}+l \bar{\beta}_{2} S_{1} I_{1}\right]-\left[l I_{1}+p l^{\prime}(1-\lambda) S_{1}+p l\left(1-\lambda_{i d}\right) I_{1}\right.} \\
& \left.+p c S_{1}+p F_{2}+(1-p) l\left(1-\lambda_{i d}\right) I_{1}+(1-p) l \beta_{2}^{L} S_{1} I_{1}\right] .
\end{aligned}
$$

After rearranging the terms and using the fact that $\bar{\beta}=p \beta_{2}^{H}+(1-p) \beta_{2}^{L}$, we obtain

$$
Q=-p S_{1}\left[l^{\prime}(1-\lambda)+c+F_{2} / S_{1}-l \beta_{2}^{H} I_{1}\right]
$$

When $\bar{\beta}_{2} \leq \hat{\beta}_{2}$, the quasi-option value satisfies

$$
\begin{aligned}
Q= & {\left[l I_{1}+l\left(1-\lambda_{i d}\right) I_{1}+l^{\prime}(1-\lambda) S_{1}+c S_{1}+F_{2}\right]-\left[l I_{1}+p l^{\prime}(1-\lambda) S_{1}+p l\left(1-\lambda_{i d}\right) I_{1}\right.} \\
& \left.+p c S_{1}+p F_{2}+(1-p) l\left(1-\lambda_{i d}\right) I_{1}+(1-p) l \beta_{2}^{L} S_{1} I_{1}\right] .
\end{aligned}
$$

After rearranging the terms, we have thus

$$
Q=(1-p) S_{1}\left[l^{\prime}(1-\lambda)+c+F_{2} / S_{1}-l \beta_{2}^{L} I_{1}\right] .
$$

\section{Appendix B}

We define the critical DR, $\hat{\beta}_{1}$, such that animal health authorities are indifferent between vaccinating in the first and second periods. Given equations (7), this value $\hat{\beta}_{1}$ satisfies $C^{*}(1)=C^{*}(0)$ with $\bar{\beta}_{2} \geq \hat{\beta}_{2}$, which implies

$$
\hat{\beta}_{1} \equiv \frac{1}{I_{0}} \frac{l^{\prime}(1-\lambda)+c \lambda-\left(F_{2}-F_{1}\right) / S_{0}}{\left\{l-c+l\left(1-\lambda_{i d}\right)-l^{\prime}(1-\lambda)\right\}} \text { with } \hat{\beta}_{1} \geq 0 .
$$


The denominator of the above expression is positive because the cost of vaccination is small compared with the periodical infection losses, $l>c$, the probability that an infected herd dies is higher than the probability that an immune herd dies, $\lambda_{i d} \geq \lambda$, and the losses as a result of infection are higher than the losses caused by immunity, $l^{\prime} \leq l$. DRs have been observed to decrease over time (Miller, 1979), as a result of disease control effects and increased awareness amongst stock owners. This implies that $\beta_{1}>\bar{\beta}_{2}$. If the campaign of vaccination in period 2 is less expensive and thus more efficient than the SO strategy, i.e. $\bar{\beta}_{2}>\hat{\beta}_{2}$, we have the following inequalities:

$$
\beta_{1} I_{0}>\bar{\beta}_{2} I_{1}>\frac{l^{\prime}(1-\lambda)+c+F_{2} / S_{1}}{l}>\frac{l^{\prime}(1-\lambda)+c \lambda-\left(F_{2}-F_{1}\right) / S_{0}}{l-c+\left[l\left(1-\lambda_{i d}\right)-l^{\prime}(1-\lambda)\right]} .
$$

The first inequality comes from the fact that $\beta_{1}>\bar{\beta}_{2}$ and $I_{1}=I_{0}\left(1-\lambda_{i d}+\beta_{1} S_{0}\right)<I_{0}$ if the strategy SO is effective, i.e. $\lambda_{i d}$ is close to unity. The second inequality occurs because the expected second-period DR is assumed greater than the critical one. The last inequality holds if vaccination costs $c$ are low enough. The first and last terms of these inequalities allow us to prove that $\beta_{1} \geq \hat{\beta}_{1}$. Consequently, we can conclude that if the campaign of second-period vaccination is optimal, then it is optimal to vaccinate the susceptible herds during the first period.

It should be noted that if the difference between the fixed vaccination costs $\Delta F=F_{2}-F_{1}$ is large enough, the critical DR $\hat{\beta}_{1}$ does not exist and the second-period vaccination programme is always dominated by the first.

\section{Appendix C}

The size and impact of FMD epidemics under optimistic and pessimistic scenarios are shown in Tables A1 and A2 respectively.

Table A1. Size and impact of FMD epidemics under the optimistic scenario, by control strategy

\begin{tabular}{lccr}
\hline & SO & SOEV & SOLV \\
\hline Duration (weeks) & 11.5 & 5.5 & 6.5 \\
Number of affected herds & 222 & 159 & 179 \\
Number of vaccinated herds & 0 & 1434 & 1827 \\
Percentage of region affected & 8 & 4 & 5 \\
Direct costs (mFF ${ }^{1}$ ) & 318 & 949 & 1181 \\
French government's subsidy (mFF) & 66 & 231 & 288 \\
EU subsidy (mFF) & 100 & 347 & 431 \\
Regional losses ${ }^{2}$ (mFF) & 839 & 1299 & 1553 \\
$\quad$ Breeding sector & 909 & 1376 & 3089 \\
$\quad$ Agricultural sector & & & \\
$\quad$ Meat and milk manufacturing & 1064 & 1196 & \\
$\quad$ Breton economy as a whole & 2229 & 2859 & \\
& & & \\
${ }^{1}$ Million French francs. & & & \\
${ }^{2}$ Net of the French government and EU subsidies. & & & \\
${ }^{3}$ Includes breeding sector. \\
SO, stamping out of infected herds; SOEV, stamping out of infected herds plus early vaccination in the control \\
area and in the ring vaccination zone; SOLV, stamping out of infected herds plus late vaccination in the control \\
area and in the ring vaccination zone.
\end{tabular}


Table A2. Size and impact of FMD epidemics under the pessimistic scenario, by control strategy

\begin{tabular}{|c|c|c|c|}
\hline & SO & SOEV & SOLV \\
\hline Duration (weeks) & 39.5 & 6.0 & 7.0 \\
\hline Number of affected herds & 554 & 187 & 227 \\
\hline Number of vaccinated herds & 0 & 1406 & 1779 \\
\hline Percentage of region affected & 34 & 4 & 5 \\
\hline Direct costs $\left(\mathrm{mFF}^{1}\right)$ & 1149 & 968 & 1214 \\
\hline French government's subsidy (mFF) & 176 & 236 & 296 \\
\hline EU subsidy (mFF) & 263 & 355 & 445 \\
\hline \multicolumn{4}{|l|}{ Regional $\operatorname{losses}^{2}(\mathrm{mFF})$} \\
\hline Breeding sector & 2216 & 1323 & 1504 \\
\hline Agricultural sector ${ }^{3}$ & 2364 & 1402 & 1586 \\
\hline Meat and milk manufacturing & 2282 & 1217 & 1260 \\
\hline Breton economy as a whole & 5194 & 2912 & 3149 \\
\hline
\end{tabular}

